\title{
Différenciation et compétitivité : quelle performance pour les exploitations viticoles françaises sous signe de qualité ?
}

\author{
Mélisande Gillot, Hélène Blasquiet-Revol(i) et Philippe Jeanneaux* (D) \\ Université Clermont Auvergne, AgroParisTech, INRAE, VetAgro Sup, UMR Territoires, 63170 Aubière, France
}

\begin{abstract}
Résumé - Les politiques de labellisation (Appellation d'origine protégée [AOP], Agriculture biologique [AB]) sont censées accroître le revenu des producteurs. Mais le revenu des agriculteurs n'est pas automatiquement amélioré par l'adoption d'une stratégie de différenciation. Nous analysons les performances économiques et techniques des exploitations ayant cette stratégie en évaluant leur efficacité technique pure et prix à l'aide de la méthode de Data Envelopment Analysis en 2014 et 2015. Nous utilisons les données du Réseau d'information comptable agricole et montrons que les exploitations en appellation d'origine protégée ont des scores d'efficacité plus faibles que celles n'ayant pas adopté ce signe de qualité. L'efficacité est variable selon les bassins viticoles. Il ressort par ailleurs que les exploitations en agriculture biologique ont des efficacités inférieures aux exploitations conventionnelles sur la période étudiée.
\end{abstract}

Mots clés : viticulture / signe de qualité / agriculture biologique / appellation d'origine protégée / efficacité économique / Data Envelopment Analysis / différenciation

\begin{abstract}
Differentiation competitiveness: What is the performance of the French label-based winegrowers? Labeling policies (Protected Designation of Origin [PDO]; Organic Farming) are supposed to increase farmers' income even if income is not automatically improved by the adoption of a strategy of differentiation. Are the adoption choices of a PDO or an Organic Farming label in viticulture economically relevant? We analyze the technical and price performance of wine-growing farms that have chosen a strategy of differentiation by evaluating economic efficiency using Data Envelopment Analysis. We used French vineyards data from the Farm Accountancy Data Network for 2014 and 2015. We show that PDO farms have lower efficiency scores than those that did not adopt this quality label. Efficiency varies according to the wine-growing regions. It also appears that organic farms have lower efficiencies than conventional farms for the period of study.
\end{abstract}

Keywords: winegrowing / quality label / organic farming / protected designation of origin / effectiveness / Data Envelopment Analysis / differentiation

\section{Introduction}

En France, la viticulture est un secteur majeur de l'économie agricole. 85000 exploitations ont des vignes, et parmi elles 68000 sont spécialisées en viticulture. Elles produisent, chaque année, environ 45 millions d'hectolitres sur 790000 hectares. Le secteur viticole représente le second secteur à l'export français avec environ 12,5 milliards d'euros de ventes annuelles, en croissance (FranceAgrimer, 2019). Même si le secteur est dynamique, il semble que les nouveaux leviers de différenciation des vins français sur un marché

\footnotetext{
$\overline{\text { *Auteur de correspondance }}$ :

philippe.jeanneaux@vetagro-sup.fr
}

mondial très concurrentiel ne sont plus seulement fondés sur les signes historiques de qualité par l'identification de l'origine et la mise en avant du terroir.

On compte 361 Appellations d'origine contrôlée ou Appellations d'origine protégée (AOC/AOP) et 74 Indications géographiques protégées (IGP) dans le secteur des vins, ce qui représentent plus de $90 \%$ de la production française commercialisée en volume.

Par ailleurs, c'est assez récemment, en mars 2012, après parution au Journal officiel européen L71 du 9 mars 2012, que la dénomination « vin biologique » est entrée en application. Le cahier des charges de cette dénomination couvre l'ensemble du processus, de la viticulture biologique à la vinification. Malgré la relative nouveauté de cette reconnaissance institutionnelle, 
le marché du vin en agriculture biologique $(A B)$ est en pleine expansion en France. En 2015, le chiffre d'affaires de la filière vin $\mathrm{AB}$ était de 670 millions d'euros pour 5186 exploitations, soit $9 \%$ du vignoble national, et pour 78665 hectares de surfaces certifiées (Agence Bio, 2019). En 2020, 9784 exploitations, soit $17,3 \%$ du vignoble national (surfaces certifiées et surfaces en conversion), étaient en agriculture biologique pour 137442 hectares de surfaces certifiées (Agence Bio, 2021).

Une des finalités assignées aux politiques de labellisation (AOP, AB...) est d'accroître le revenu des producteurs en s'appuyant sur la compétitivité hors coût, notamment dans le cadre de stratégies de différenciation collectives. La stratégie de différenciation vise à obtenir un avantage concurrentiel en proposant un produit à forte valeur ajoutée à une cible large. Elle diverge de deux autres stratégies: la stratégie de domination par les coûts, qui vise à obtenir un avantage concurrentiel en proposant un produit à faible coût à une cible stratégique large, avec de faibles marges mais des volumes importants; et la stratégie de focalisation sur un segment de marché spécifique (Porter, 1985). L'Institut national de la statistique et des études économiques (INSEE), dans une de ses publications (Dedieu et al. 2017), considère que l'AB améliore la performance des exploitations agricoles, et en particulier viticoles. C'est un des rares travaux sur la performance des exploitations viticoles, tous bassins de production français confondus. Par ailleurs, Guyomard (2013), dans le rapport de l'Institut national de la recherche agronomique (INRA) «Vers des agricultures à hautes performances », avait souligné le peu de travaux existants sur les performances économiques de l'agriculture biologique. Le rapport (p. 71) regrettait qu'il soit "impossible, sur la base de la revue de la littérature, de dégager une conclusion claire, simple et générale quant à des performances économiques supérieures ou au contraire inférieures des exploitations en agriculture biologique versus en agriculture conventionnelle ».

Devant le peu de travaux d'évaluation des impacts des signes de qualité sur la performance économique des exploitations viticoles, cet article propose une contribution originale sur la performance des exploitations viticoles selon le choix d'un signe de qualité (AOC/AOP, IGP, AB). L'article analyse et compare les performances économiques et techniques des exploitations ayant fait le choix de la différenciation par l'AB et l'AOC/AOP ou encore l'IGP. La question principale est de savoir si le choix de l'adoption de l'AB, de l'AOP ou de l'IGP en viticulture est économiquement pertinent.

Dans un premier temps, nous présentons le cadre méthodologique de cette recherche (2). Ensuite, nous exposons les résultats concernant la performance technique et économique des exploitations viticoles françaises pour les années 2014 et 2015 avec un point particulier fait sur l'AB (3). Nous discutons des résultats (4) avant de conclure (5).

\section{Matériels et méthodes}

\subsection{La méthode Data Envelopment Analysis pour analyser la performance technique et économique}

Nous mesurons la performance technique et économique des exploitations viticoles en évaluant l'efficacité technique pure et l'efficacité prix des facteurs de production à l'aide de

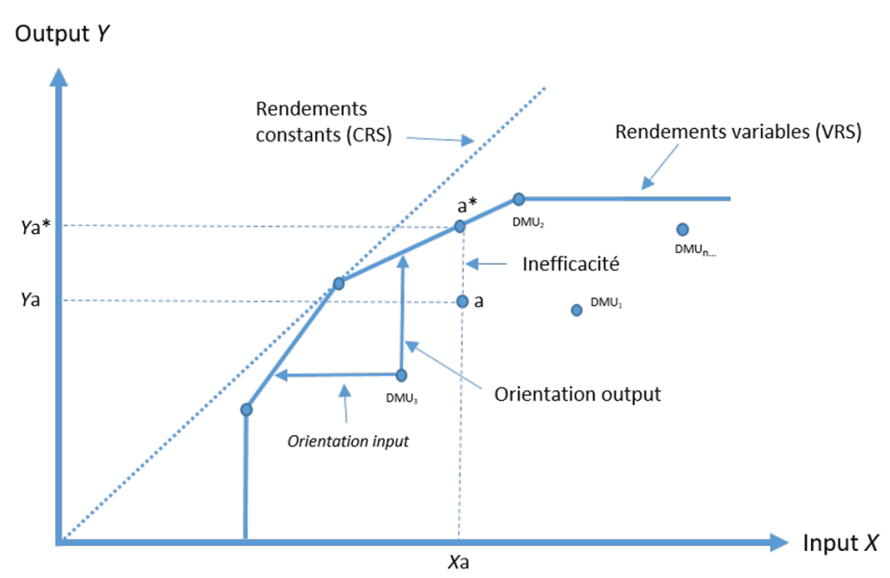

Fig. 1. Frontière de production et mesure de l'efficacité dans l'orientation output.

Fig. 1. Production frontier and output-oriented measure of efficiency.

l'approche dite de Data Envelopment Analysis (DEA). La DEA permet de mesurer l'efficacité totale de l'activité de l'exploitation plutôt que de calculer des productivités partielles de chacun des facteurs de production (inputs). D'un point de vue sémantique, dès lors que nous considérons l'existence de facteurs extérieurs ne pouvant pas être pris en compte, il est d'usage d'utiliser le terme efficacité. C'est donc le terme efficacité qui sera utilisé, en se référant à la notion d'efficience.

Nous développons une analyse non paramétrique de l'efficacité d'un ensemble d'observations par enveloppement des données (DEA). Au départ, Farrell (1957) proposa le concept d'efficacité et sa mesure avant que la DEA soit introduite pour la première fois par Charnes et al. (1978). Ils proposèrent un modèle de programmation mathématique capable d'évaluer l'efficacité relative d'un ensemble d'entités comparables, appelées unités de prise de décision (Decision Making Units, DMU), avec de multiples entrées et sorties. Ils ont posé le concept d'ensemble des technologies, qui correspond à l'ensemble de plans de production (input $\mathrm{X}$, output $\mathrm{Y}$ ) tels qu'il est possible, technologiquement, de produire l'output Y avec l'input X. Une fois défini l'ensemble des technologies, le calcul des efficacités se fait par un calcul d'optimisation. Ici, les observations qui sont les plus efficaces et qui formeront la frontière d'efficacité sont celles qui maximisent la production en maintenant constantes leurs ressources factorielles. La DEA utilisée ici est une approche non paramétrique qui permet de ne pas avoir besoin de spécifier une forme fonctionnelle entre les intrants (input X) et la production (output Y). D'autres approches existent que l'on peut retrouver dans la littérature sur des méthodes DEA stochastiques, par exemple chez Olesen et Petersen (2016). Dans l'approche que nous utilisons, les entreprises les plus efficientes forment une frontière d'efficacité qui enveloppe les entreprises les moins performantes, et dont la distance par rapport à la frontière est interprétée comme une mesure de l'inefficacité. Les exploitations situées sur la frontière servent de référence ou «benchmark» aux exploitations moins efficaces (Fig. 1).

Nous faisons l'hypothèse que les inputs et les outputs sont supposés de qualité équivalente pour toutes les exploitations viticoles qui sont censées avoir une technologie de production 
Tableau 1. Année 2014 (et 2015) : Part du raisin dans le produit viticulture/viniculture.

Table 1. Year 2014 (and 2015): Share of grapes in the viticulture/viniculture product.

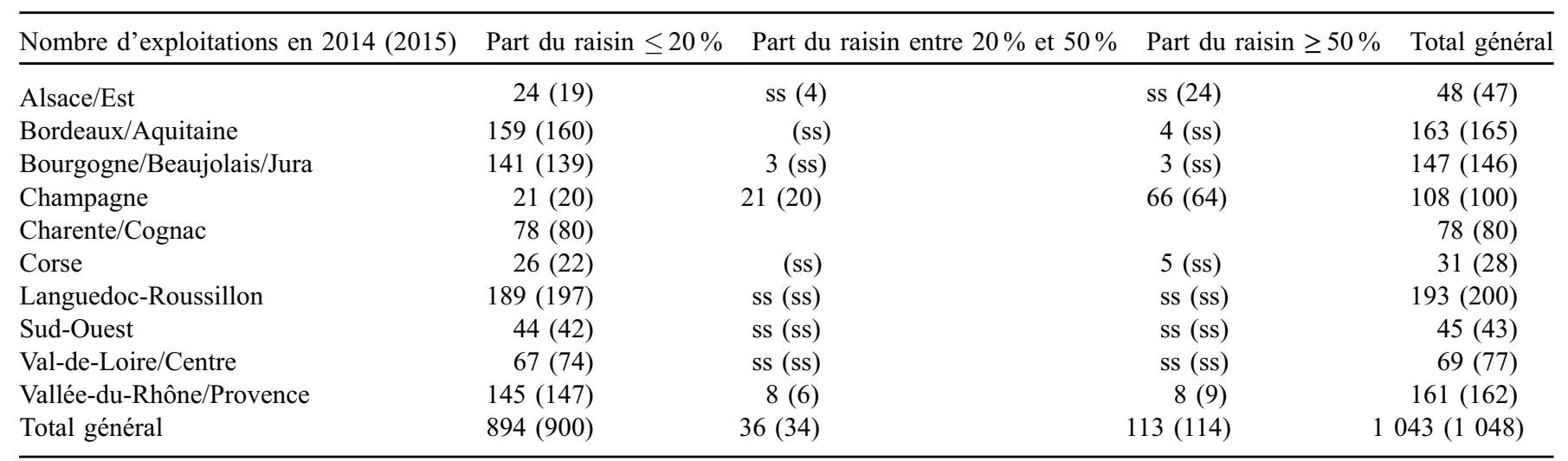

ss : Secret statistique-Les effectifs pour l'année 2015 sont entre parenthèses.

commune (Bogetoft, 2012). Toutefois, nous avons mesuré les efficacités pour les exploitations conventionnelles et les exploitations en agriculture biologique pour discuter l'hypothèse d'une différence de technologie comme l'avaient proposé Oude Lansink et al. (2002). Les mesures d'efficacité par bassin de production permettent également d'identifier la performance des exploitations d'un même bassin, dont on suppose qu'elles utilisent la même technologie. Nous avons réalisé quatre analyses DEA : une analyse pour les exploitations en $\mathrm{AB}$, une pour les exploitations en production conventionnelle, et cela pour les années 2014 et 2015. Nous avons par ailleurs restreint l'analyse aux seuls systèmes qui vinifient tout ou partie de leur récolte pour comparer des exploitations ayant des technologies très proches.

Concernant les hypothèses techniques, nous avons choisi une orientation output qui correspond à la maximisation des outputs pour un niveau d'inputs donné (Briec et Peypoch, 2010). Les exploitations en IGP, AOC/AOP ou AB, dont le fonctionnement repose sur une stratégie de différenciation, sont censées augmenter les outputs en valeur (en euros) pour un même niveau d'inputs, dans la mesure où elles tentent de capter plus de valeur ajoutée grâce à un consentement à payer supérieur du consommateur par rapport aux vins «standards ». La fonction de distance utilisée ne varie pas. Il s'agit d'une fonction de distance directionnelle dite à la Shephard (1981). L'hypothèse de rendement d'échelle variable (VRS) a été retenue pour toutes les productions. Les exploitations qui ont une efficacité optimale ont un score égal à $1\left(\mathrm{DMU}_{2}\right.$ sur la Fig. 1). En orientation output, plus l'exploitation a un score supérieur à 1 et moins elle est efficace $\left(\mathrm{DMU}_{1}\right.$ ou $\mathrm{DMU}_{3}$ sur la Fig. 1).

Enfin, l'originalité de l'approche est de réaliser le travail en deux étapes :

Dans une première étape, nous ne tenons pas compte des prix des outputs et des inputs afin d'évaluer l'efficacité technique pure (en volume). Cette première mesure permet d'évaluer les capacités purement techniques du viticulteur. Mais ces choix techniques peuvent résulter des conditions à respecter pour valoriser les produits. Autrement dit, une inefficacité technique peut résulter de l'application d'un cahier des charges qui contraint l'obtention d'un rendement par hectare.
C'est pourquoi, dans une deuxième étape, nous introduisons les prix réels des outputs pour prendre en compte les spécificités de la compétitivité hors coût, permettant ainsi de tenir compte de la capacité des exploitations agricoles à capter la valeur. Il s'agit en fait dans un deuxième temps d'une mesure de l'efficacité "prix». Dans cette deuxième étape, les inputs restent en valeur physique. C'est pourquoi nous ne calculons pas une efficacité allocative qui intégrerait les prix des inputs et des outputs. Il s'agit de la notion dite «revenue efficiency», pour laquelle les inputs sont mesurés en termes physiques et les outputs en termes monétaires (Sahoo et al., 2014). Nous avons, par ailleurs, comparé les scores d'efficacité des exploitations viticoles en AB, IGP et AOC/AOP en prenant en considération leur appartenance aux différents bassins viticoles français. Nous avons également réalisé ces mesures pour les exploitations conventionnelles afin d'avoir une idée de la différence en matière de compétitivité de la stratégie de différenciation des exploitations sous IGP, AOP ou AOC.

\subsection{Données mobilisées}

Nous avons utilisé les données du Réseau d'information comptable agricole (RICA), dont le champ d'observation comprend les exploitations agricoles dites moyennes ou grandes (production brute standard [PBS] supérieure à $25000 €)$. Nous avons considéré les exploitations des OTEX 3511 (AOP/AOC), 3512 (IGP), 3513 (AOP et IGP), 3520 (vins et autres vins de qualité) et 3540 (autres vignobles). Ces échantillons correspondent à 1055 exploitations pour l'année 2014 et 1073 exploitations pour l'année 2015 dans le RICA. Ils ont été réduits à respectivement 1043 et 1048 exploitations pour réaliser les traitements de Data Envelopment Analysis (DEA). Ce tri, fondé sur les variables de produits bruts (sommes des ventes, variations de stocks et autoconsommation) pour les codes correspondants aux différents produits viticoles et vinicoles, a permis d'écarter les exploitations ne produisant pas de produits viticoles ou vinicoles. Néanmoins, dans cet échantillon, les exploitations peuvent être productrices de raisin pour vin et/ou de vin (Tab. 1). Au final, pour 2014 (et pour 2015), l'échantillon traité rassemble 930 (934) exploitations produisant du vin et exclut les 113(114) 


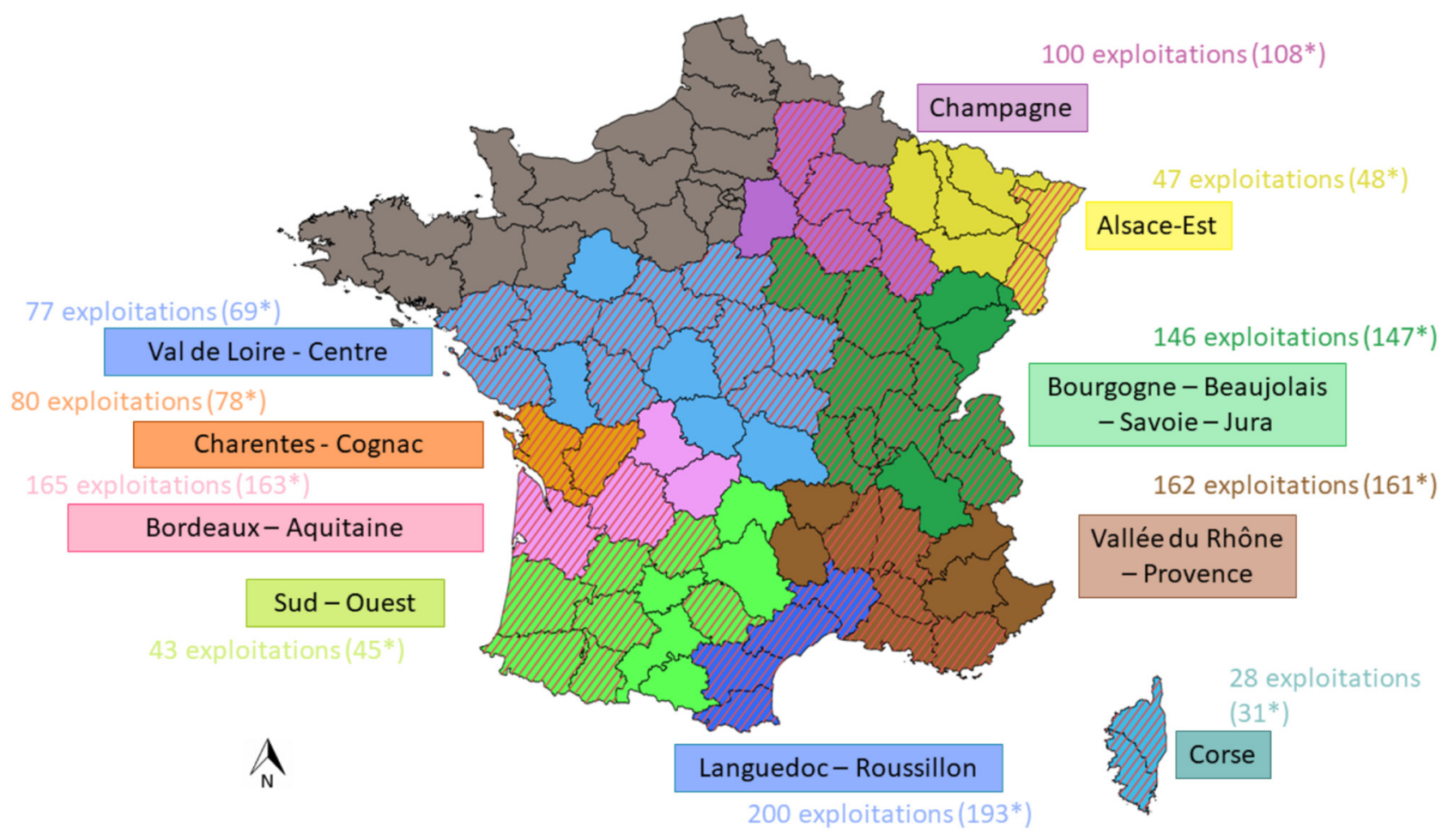

Fig. 2. Répartition géographique des exploitations agricoles des échantillons constitués (2015) (*: effectif 2014). Source: Traitements Rica 2015 des auteurs.

Fig. 2. Geographical distribution of farms in the samples (2015) (*: number of farms in 2014).

exploitations dont la part de raisin dans le produit en vitiviniculture est supérieure à $50 \%$ (Tab. 1). Ce choix permet de mener l'analyse à technologie similaire entre OTEX et bassins de production. Les exploitations dont la part de raisin dans le produit viticole est supérieure à $50 \%$ sont principalement présentes dans le bassin viticole «Champagne».

Les zones d'appellation dans lesquelles sont situées les exploitations sont connues et se réfèrent aux aires géographiques des AOC/AOP de l'Institut national de l'origine et de la qualité (INAO). Les exploitations ont été appariées avec le code INSEE de la commune d'appartenance du siège de l'exploitation, ce qui a permis de mesurer la performance par bassin de production (Tab. 2). Sur l'échantillon de l'année 2015, 71\% des exploitations sont dans l'OTEX 3511, i.e., engagées dans des démarches AOC/AOP et 7,9\% sont dans l'OTEX 3513 (AOP et IGP). La part des exploitations en agriculture biologique (ou en conversion) dans l'échantillon est de $11,3 \%$, ce qui représente $8,6 \%$ des surfaces et correspondait aux données de l'Agence Bio (8,7\% des surfaces de vignes en agriculture biologique) en 2015. Les exploitations engagées en $\mathrm{AB}$ dans notre échantillon sont à plus de $90 \%$ également en AOC/AOP (OTEX 3511). La répartition des exploitations en fonction des OTEX et des modes de production est quasiment identique pour les années 2014 et 2015 . Le tableau 2 ne présente que les résultats de l'année 2014, qui restent très proches de ceux de l'année 2015.

De manière générale (Tab. 3), on remarquera que les exploitations qui produisent des vins AOC/AOP ont une surface agricole utile plus petite. Elles sont aussi plus spécialisées que celles produisant des vins IGP ou des vins sans label officiel de qualité, mais leur surface en vigne reste inférieure aux autres systèmes. Les exploitations AOP/AOC spécialisées utilisent un peu plus de main-d'œuvre, et ont une productivité du travail plus faible par hectare. Elles ont aussi des rendements plus faibles par hectare qui sont compensés par des prix plus élevés du vin. Ces exploitations en AOP/AOC ont au final des produits totaux plus élevés que les autres exploitations et de meilleurs soldes de gestion par hectare (excédent brut d'exploitation et résultat courant). Autrement dit, ces exploitations concentrent plus de produit et de richesse par hectare. Elles concentrent également plus de capital productif (actif du bilan) que les autres. Enfin, il ressort que les exploitations en agriculture biologique ont des caractéristiques assez similaires aux exploitations AOP/AOC spécialisées. Le tableau S1 «Description de l'échantillon d'études pour l'année 2014 (Données RICA)» est consultable en matériel supplémentaire.

\subsection{Choix de l'année et de la distribution géographique}

Le choix des années d'études s'est porté sur les années 2014 et 2015, dernières années disponibles des données du RICA au moment des traitements. La campagne 2015 a été marquée par une période de canicule, dans l'ensemble des bassins de production (CCVF, 2015). La majorité des interprofessions a déploré de faibles volumes. Nous avons donc complété l'étude de l'année 2015 par celle de l'année 2014, afin de se détacher d'un potentiel effet «année». 
M. Gillot et al. : Cah. Agric. 2022, 31, 2

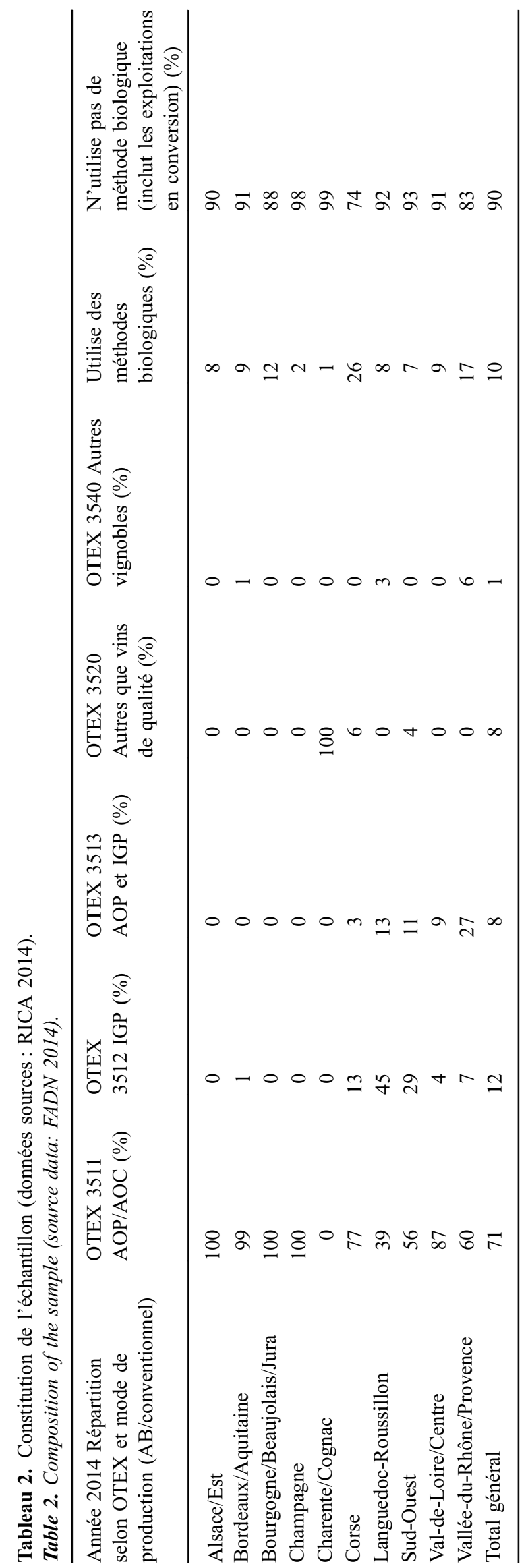

Page 5 de 12 
M. Gillot et al. : Cah. Agric. 2022, 31, 2

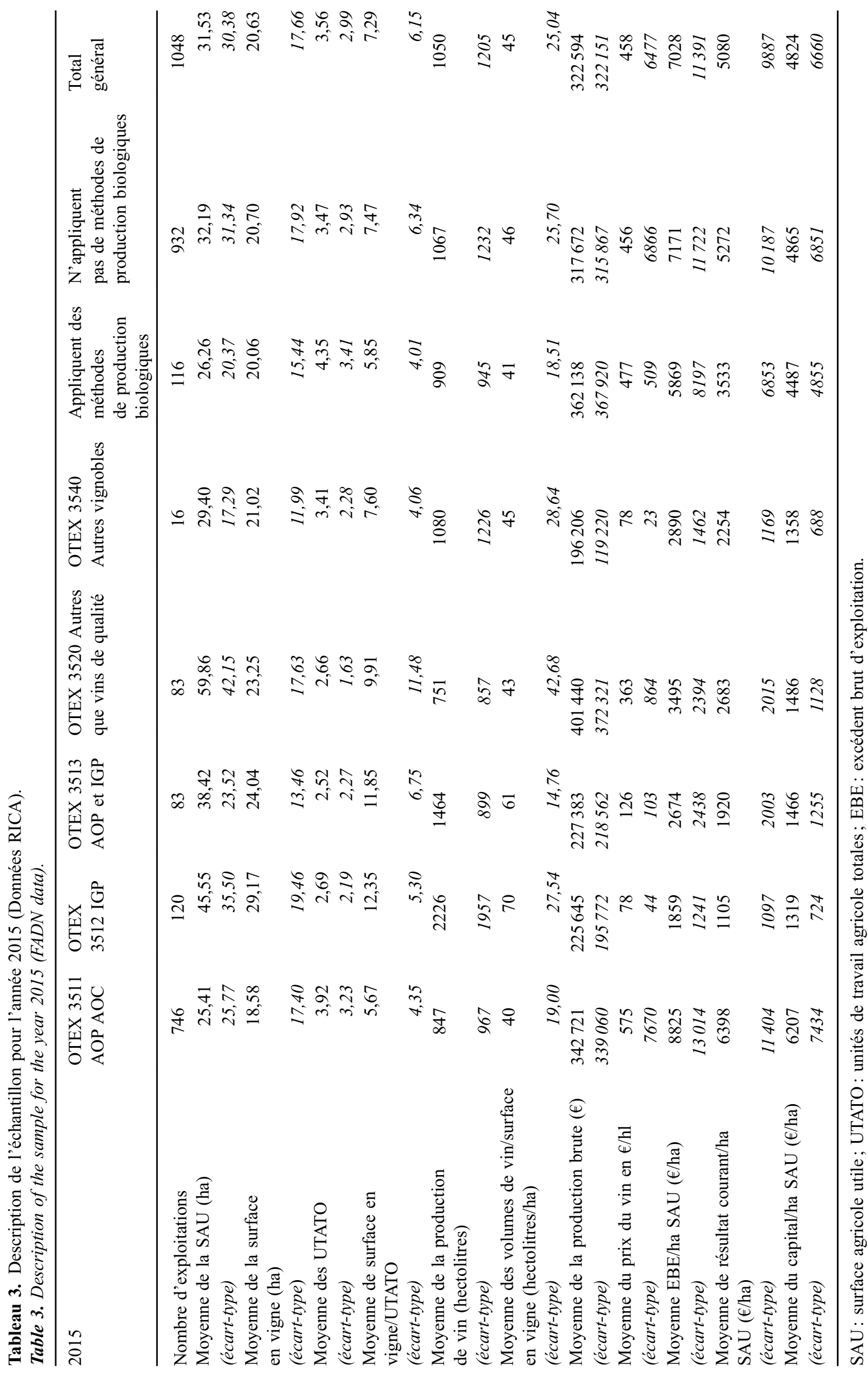




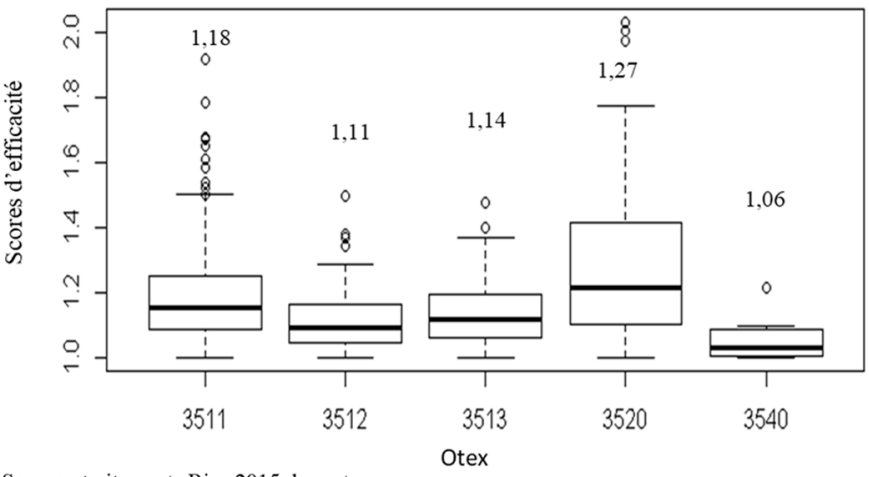

Source : traitements Rica 2015 des auteurs.

Fig. 3. Scores d'efficacité «technique pure» en fonction des OTEX (2015) pour les exploitations à dominante «vin». Source: Traitements Rica 2015 des auteurs.

Fig. 3. "Pure technical" efficiency scores according to OTEX (2015) for wine farms.

Pour une meilleure lisibilité des résultats et afin de respecter le secret statistique, nous avons "agrégé» les résultats à l'échelle de grands bassins viticoles. La figure 2 présente la répartition géographique de l'échantillon d'étude. Les départements dans lesquels les exploitations sont présentes sont hachurés en rouge. Par exemple: dans le bassin dit Alsace-Est, les exploitations se répartissent dans les départements du Bas-Rhin et du Haut-Rhin.

\subsection{Spécification du modèle}

Nous avons utilisé cinq «inputs» pour le calcul de l'efficacité technique pure : (1) le foncier, pris en compte par la surface agricole utile (SAU en hectares); (2) le travail, pris en compte par le nombre d'unités de travail agricole totales (UTA) sur l'exploitation (salariés et non-salariés); (3) les charges opérationnelles, qui comprennent les engrais et amendements, les produits phytosanitaires et l'eau d'irrigation; (4) les consommations intermédiaires, qui rassemblent les achats de carburants, lubrifiants, eau, gaz, électricité ; (5) les charges liées à l'appareil de production, correspondant aux achats de travaux et services extérieurs, le crédit-bail, les charges d'entretien et d'amortissement des bâtiments et des matériels, ainsi que l'amortissement des aménagements des plantes pérennes. Du côté des «outputs», pour le calcul de l'efficacité technique pure, nous avons pris en compte le vin produit en quantité (hectolitres), le raisin produit en quantité (hectolitres) et les autres produits végétaux (en quantité [hectolitres] et en euros). Pour le calcul de l'efficacité " prix », nous avons pris en compte le vin, le raisin produit et les autres produits végétaux en valeur (euros).

\section{Résultats}

\subsection{Mise en contexte et tour d'horizon des performances des exploitations viticoles françaises}

Dans un premier temps, nous avons calculé des scores d'efficacité hors prix pour avoir une première estimation de l'efficacité technique pure des exploitations viticoles (Fig. 3). Les résultats observés en 2015 sont similaires à ceux de 2014 .

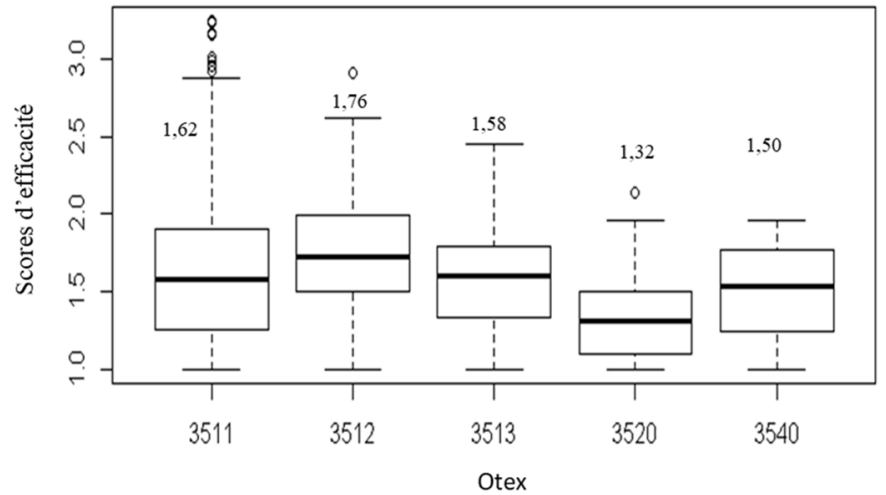

Source : traitements Rica 2015 des auteurs.

Fig. 4. Scores d'efficacité " prix » en fonction des OTEX (2015) pour les exploitations à dominante «vin». Source : Traitements Rica 2015 des auteurs.

Fig. 4. Price efficiency scores according to OTEX (2015) for wine farms.

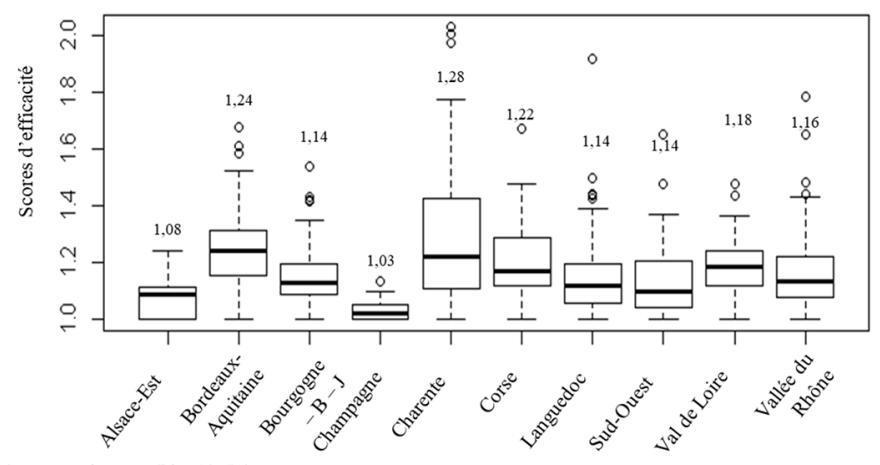

Fig. 5. Score d'efficacité technique pure des exploitations conventionnelles à dominante vin, selon les bassins viticoles (2015). Source: Traitements Rica 2015 des auteurs.

Fig. 5. Pure technical efficiency score of conventional wine farms by wine growing areas (2015).

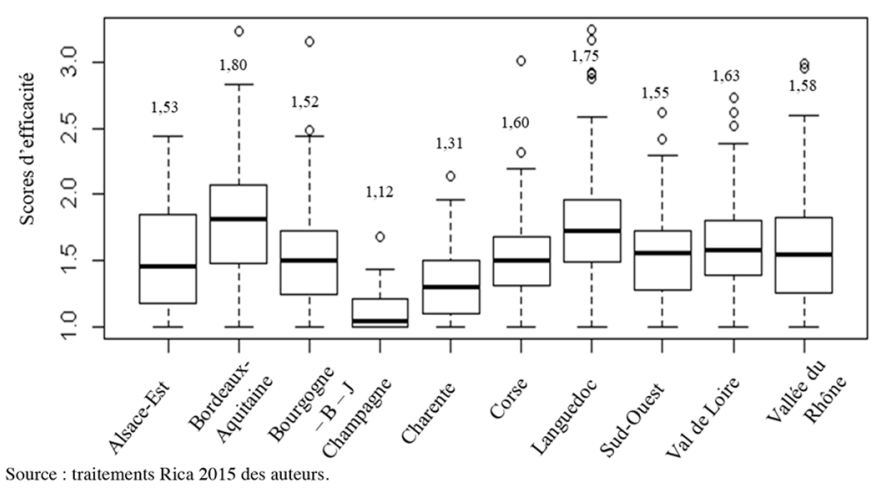

Fig. 6. Score d'efficacité prix des exploitations conventionnelles à dominante vin, selon les bassins viticoles (2015).

Fig. 6. Price efficiency score of conventional wine farms by winegrowing regions (2015). 
Tableau 4. Score d'efficacité technique pure et prix des exploitations à dominante vin selon les bassins viticoles - Année 2014 (traitements des auteurs).

Table 4. Pure technical efficiency score and prices of wine farms by wine-growing regions - Year 2014 (authors' treatments).

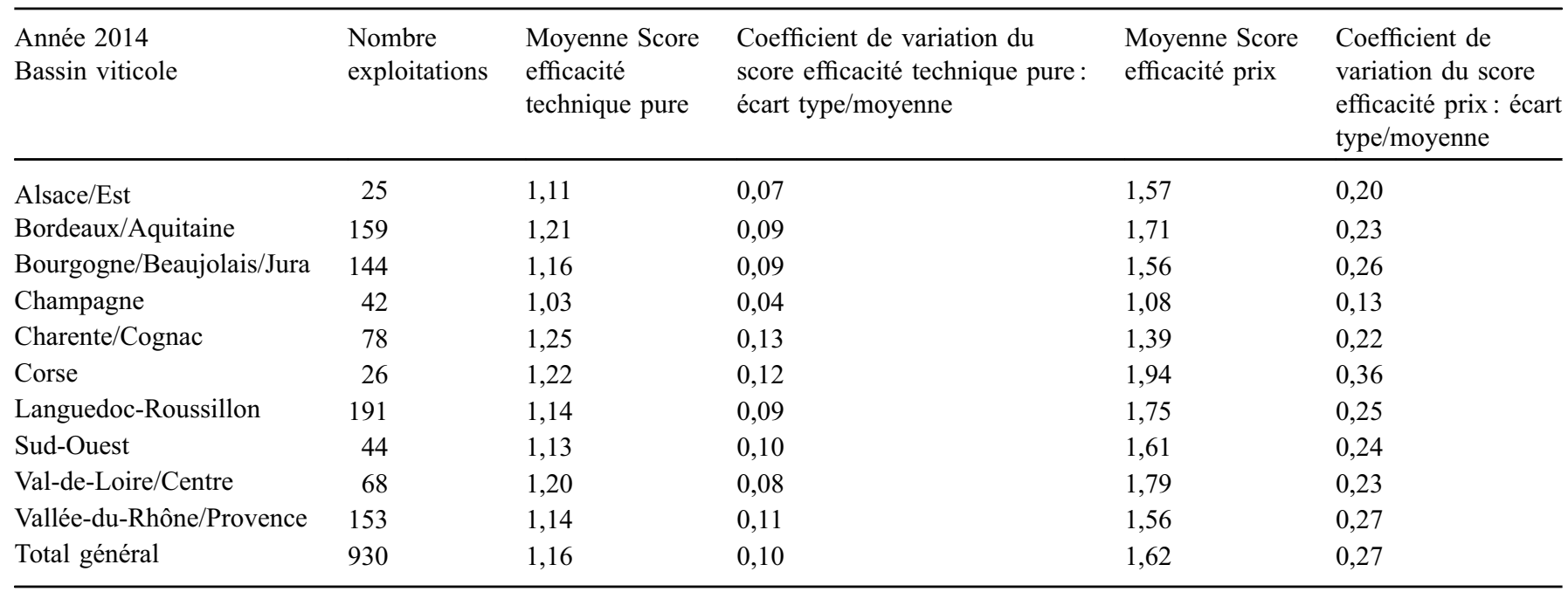

Pour 2015, il ressort que les systèmes les plus efficaces sont les quelques exploitations qui ne sont pas soumises à un cahier des charges AOP/AOC (score=1,06 sachant que l'efficacité optimale correspond à un score égal à 1). Autrement dit, les stratégies sans lien fort et formel au terroir et/ou plutôt dans une logique de valorisation de la notion de cépage ont la meilleure capacité à maximiser les produits pour un niveau d'inputs donné. Les exploitations viticoles en AOP/AOC sont moins performantes techniquement $($ score $=1,18)$ car les pratiques de production sont, d'une part, plus strictes et plus coûteuses, et d'autre part, contraignent les volumes du fait de l'existence de limites de rendement à l'hectare.

Dans un second temps, nous avons intégré les prix réels des produits (Fig. 4). Nous avons constaté que la hiérarchie des résultats constatée pour l'efficacité technique pure changeait peu, à l'exception des vins IGP (3512) et des autres vins de qualité (3520). Il ressort que le prix plus élevé des vins AOC/ AOP ne parvient pas vraiment à infléchir la tendance observée «hors prix ». En première analyse, les systèmes productifs en AOC/AOP ainsi qu'en IGP ont une moindre efficacité prix, contrairement aux systèmes sans indications géographiques. On notera le cas particulier de l'Otex 3520 «Vins et autres vins de qualité », qui correspond majoritairement aux exploitations produisant des vins et spiritueux des bassins Charentes-Cognac et Armagnac, et qui améliorent considérablement leur efficacité prix quand on intègre les prix réels dans le calcul des outputs. La forte inefficacité technique pure de ces systèmes, relevée lors de l'analyse «hors prix», traduit la stratégie des viticulteurs de « faire du volume pour la distillation » en utilisant de très fortes quantités d'intrants. Puis, l'excellente valorisation du Cognac sur le marché export compense très largement l'inefficacité technique pure de départ.

\subsection{Des efficacités variables selon les bassins viticoles}

Nous avons analysé les scores d'efficacité des exploitations viticoles selon leur localisation dans les différents bassins viticoles et identifié quatre catégories de bassins selon leur efficacité (Fig. 5 et 6 pour 2015 et Tab. 4 pour 2014).

Tout d'abord, il ressort que les bassins les plus efficaces techniquement sont l'Alsace-Est et la Champagne, tant pour 2015 que pour 2014. Ces deux bassins se distinguent par leurs meilleures efficacités techniques pures (score de 1,08 à 1,11 pour l'Alsace et 1,03 pour le bassin Champagne en 2014 et 2015) (Fig. 5 et Tab. 4). La prise en compte des prix réels des outputs ne modifie pas la hiérarchie (Fig. 6 et Tab. 4). Le bassin champenois bénéficie d'un double avantage: des rendements autorisés en AOC/AOP Champagne élevés, à 10500 kilos de raisin par hectare, soit environ $65 \mathrm{hl} / \mathrm{ha}$, et des prix élevés.

À l'opposé, les plus mauvais scores d'efficacité technique pure sont relevés sur le bassin Bordeaux-Aquitaine et le bassin Charente-Cognac (scores respectivement à 1,24 et 1,27). Nous avons déjà présenté les spécificités du bassin Charentais et n'y revenons pas. Nous nous concentrons sur le bassin BordeauxAquitaine qui est celui qui a soulevé le plus la question d'un effet conjoncturel de l'année 2015. En effet, les scores observés en 2015 présentent une forte inefficacité technique pure $($ score $=1,24)$ et une très forte inefficacité prix (score $=$ 1,80) (Fig. 5 et 6). Le vignoble bordelais a de fait connu sur cette période des difficultés, notamment commerciales. L'AOP/AOC ne semble plus être une démarche qualité suffisante pour tirer les prix vers le haut, en dehors des crus et autres châteaux prestigieux.

On trouve ensuite un ensemble de bassins qui ont des scores d'efficacité technique pure compris entre 1,13 et 1,18 et des scores d'efficacité prix compris entre 1,50 et 1,60. Il s'agit des bassins Vallée du Rhône, Bourgogne et Sud-Ouest. Ces bassins présentent (en 2014 comme en 2015) des tendances assez similaires : des inefficacités techniques et économiques « dans la moyenne» (Fig. 5 et 6 et Tab. 4). Les scores de 2015 sont similaires à ceux de 2014 .

Enfin, on trouve une quatrième catégorie qui rassemble les bassins du Languedoc et du Val de Loire-Centre et Corse. Leur efficacité technique est similaire ou légèrement en dessous de 
Tableau 5. Comparaison des scores de performance «technique» et «prix» pour les exploitations en AB et en conventionnel (2015). Table 5. Comparison of "technical" and "price" performance scores for organic and conventional wine farms (2015).

\begin{tabular}{|c|c|c|c|c|}
\hline $\begin{array}{l}2015 \text { (traitement DEA } \\
\text { sur l'ensemble des exploitations) }\end{array}$ & $\begin{array}{l}\text { Moyenne efficacité } \\
\text { «technique pure» }\end{array}$ & $\begin{array}{l}\text { Écart-type efficacité } \\
\text { «technique pure» }\end{array}$ & $\begin{array}{l}\text { Moyenne efficacité } \\
\text { «Prix » }\end{array}$ & $\begin{array}{l}\text { Écart-type efficacité } \\
\text { «Prix » }\end{array}$ \\
\hline Bio & 1,28 & 0,20 & 1,72 & 0,45 \\
\hline
\end{tabular}

la moyenne (score autour de 1,20) alors que leur efficacité prix est médiocre (score entre 1,75 et 1,94 ), comme si les prix de vente ne généraient pas suffisamment de valeur ajoutée. Le Languedoc est le bassin viticole qui a la situation la plus contrastée sur ce point. Par exemple, pour 2014, son efficacité technique pure était supérieure à la moyenne $(1,14$ contre une moyenne à 1,16$)$, mais son efficacité prix était médiocre $(1,75$ contre 1,62 pour la moyenne), situation qui révèle une difficulté de valorisation de la production (Tab. 4).

Enfin, il apparaît que la dispersion des scores au sein des bassins de production n'est pas homogène, même si elle reste contenue avec des coefficients de variation qui fluctuent entre 0,04 et 0,23 pour l'efficacité technique pure. La prise en compte des prix est une source de dispersion au sein des bassins. Par exemple pour 2014, les coefficients de variation des scores d'efficacité prix sont supérieurs de 0,1 à 0,24 . Le bassin du Champagne est le plus homogène, alors que les bassins de production Corse et Vallée du Rhône sont parmi les plus hétérogènes.

Les performances techniques et économiques plutôt moyennes des exploitations en AOC/AOP et de certains bassins de production ne sont peut-être pas étrangères à la montée en puissance de l'agriculture biologique en viticulture. En effet, les producteurs les plus compétents techniquement ont peut-être plus de capacité à mener une conversion vers l'agriculture biologique et quittent le groupe des conventionnels.

\subsection{Les performances techniques et économiques en agriculture biologique}

Les exploitations viticoles en $\mathrm{AB}$ relèvent de deux catégories dans notre échantillon:

- celles appliquant uniquement des méthodes reconnues par le cahier des charges en AB (90 exploitations en 2015) (AB2);

- celles appliquant des méthodes reconnues par le cahier des charges en $\mathrm{AB}$ ou des méthodes proches de l'AB (26 exploitations en 2015) (AB5).

Les exploitations en conversion vers l'AB (29 exploitations en 2015) ont été rattachées aux exploitations conventionnelles.

Les exploitations en viticulture conventionnelle sont les plus nombreuses dans l'échantillon avec 932 individus par rapport à l'effectif fusionné des exploitations en $\mathrm{AB}(\mathrm{AB} 2+$ AB5), qui s'élève à 116 individus.

Il ressort que les exploitations appliquant uniquement des méthodes reconnues par le cahier des charges officiel en $\mathrm{AB}$ (AB2) ne diffèrent pas des conventionnelles en matière de surface et de capital mobilisé pour produire. La surface moyenne est d'environ 20 hectares et le capital (hors foncier) autour de $4900 € /$ ha. Cependant, le rendement par hectare est différent avec une dizaine d'hectolitres en moins pour celles en AB2. On peut penser qu'il est compensé par des prix de vente du vin ou de la vendange plus élevés. C'est ce que signale le «prix» du vin, en moyenne plus important en $\mathrm{AB}$ qu'en conventionnel $(477 € / \mathrm{hl}$ contre $456 € / \mathrm{hl})$ (Tab. 3). On notera que les exploitations en agriculture biologique utilisent plus de main-d'œuvre par hectare que les exploitations en conventionnel. Une unité de travail annuelle en $\mathrm{AB}$ valorisait 4,01 ha contre 6,34 en conventionnel en 2015 (Tab. 3).

Concernant la mesure de l'efficacité technique pure et prix, l'observation principale est qu'il n'y a pas de «décrochage» important de l'un des «groupes», tant en valeur qu'en volume (Tab. 5). L'efficacité technique pure des exploitations conventionnelles (score $=1,23$ ) est comparable à celle des systèmes $\mathrm{AB}(\mathrm{score}=1,28)$. Les rendements plus faibles en $\mathrm{AB}$ sont compensés par une utilisation plus faible des inputs également. Autrement dit, la viticulture en AB n'est pas à l'origine d'une détérioration d'efficacité technique pure forte, comme elle n'est pas non plus à l'origine d'une amélioration de l'efficacité technique pure.

En revanche, une fois intégrés les prix des outputs, le score d'efficacité en AB $(1,72)$ s'est détérioré par rapport aux exploitations en conventionnel, alors que nous nous attendions à un gain d'efficacité prix causé par la meilleure valorisation du vin issu de l'AB. En fait, le surplus de prix de vente capté par les viticulteurs ne fait que compenser le « surprix » des produits biologiques de traitement et le surplus de main-d'œuvre.

La dégradation de l'efficacité prix montre qu'il est difficile d'améliorer son efficacité prix en passant d'une agriculture conventionnelle à une agriculture sans intrants de synthèse censée être plus respectueuse de la santé du producteur, du consommateur et de l'état de l'environnement. Les exploitations en $\mathrm{AB}$ auraient plus de difficultés à aligner de manière cohérente leurs pratiques de production à la valorisation sur le marché. Les résultats montrent que les choix techniques ne sont pas optima et signalent l'existence de gaspillage d'inputs et/ou l'insuffisance des rendements. Il en est de même sur le positionnement sur le marché qui montre une difficulté à mieux valoriser les vins en $\mathrm{AB}$.

Ces écarts de performance pourraient s'expliquer par des situations marquées par de possibles effets d'apprentissage techniques et commerciaux liés aux conversions récentes des exploitations conventionnelles en $\mathrm{AB}$. Les conditions pédoclimatiques ne sont peut-être pas identiques avec des exploitations en $\mathrm{AB}$ qui ont pu tenter de valoriser en $\mathrm{AB}$ des parcelles à plus faible potentiel agronomique. Il en est de même avec le contexte économique qui n'est pas nécessairement identique entre le marché des vins en $\mathrm{AB}$, conventionnels et $\mathrm{AOP} / \mathrm{AOC}$. 


\section{Discussion}

Nous proposons de mettre à la discussion deux types de résultats avant d'ouvrir sur quelques perspectives:

- les résultats moyens de la stratégie de différenciation par l'origine et le terroir (AOC/AOP) interrogent sur une nouvelle typologie de la différenciation;

- les résultats de la stratégie $A B$ montrent que le signe de qualité $\mathrm{AB}$ ne semble pas apporter de gains d'efficacité par rapport au conventionnel.

\subsection{L'AOC: un cadre trop rigide?}

Les résultats des efficacités techniques et économiques en AOC/AOP montrent que ces exploitations ont la moins bonne efficacité prix. La différenciation fondée sur l'origine et le terroir ne semble pas être la garantie de l'efficacité prix des exploitations. L'AOC est un standard de base qui positionne désormais les vins sur le marché de la grande distribution et de l'export. La part des exploitations AOC/AOP parmi les exploitations viticoles en fait une norme plutôt qu'un facteur de différenciation fort. La faible adaptabilité des cahiers des charges incite certains producteurs à sortir des AOC/AOP (Edelmann et al., 2020), parfois pour maintenir des variétés hybrides plus résistantes à certains ravageurs ou aux aléas climatiques. Cette situation est exacerbée dans le contexte actuel de dérèglement climatique.

La crise du signe AOC se traduit par une remise en cause de cette stratégie. L'Institut national de l'origine et de la qualité (INAO) avait déjà tenté de relancer ce signe officiel de la qualité et de l'origine (SIQO) en imaginant, en 2004, une nouvelle segmentation par la reconnaissance d'AOC d'excellence (Agra Presse, 2004). La tentative échoua.

Différentes stratégies sont à l'œuvre pour contourner ou compléter le signe AOC/AOP devenu trop générique. Trois stratégies ressortent:

- une stratégie « vins de cépage et vins technologiques » dans une logique d'adaptation à la concurrence internationale (Rousset et Traversac, 2014);

- une stratégie de "vins de terroir », qui viserait à valoriser plus fortement les patrimoines matériels et immatériels (sols, cépages et savoir-faire) dans une logique de distinction, d'originalité et d'excellence;

- enfin, une stratégie de vins en AB et/ou «Nature». Dans cette logique, plusieurs types de viticulteurs sont actifs et se distinguent (Pouzenc et Vincq, 2013). On trouve, tout d'abord, les opportunistes modernisateurs de l'AOC qui voient dans l'AB la possibilité d'apporter la brique environnementale qui manque à l'AOC; ensuite, les viticulteurs alternatifs, nouveaux venus et opposés au modèle conventionnel et militant pour la confection d'un vin sain; et enfin les esthètes, promoteurs des vins «nature» originaux. Ces deux derniers types ne se sentent plus soumis à l'ancrage au terroir.

\subsection{L'agriculture biologique : au-delà des changements de pratiques, une conversion globale à réaliser}

Il ressort de cette étude que les exploitations viticoles en $\mathrm{AB}$ ont une efficacité technique pure assez comparable à celle des exploitations conventionnelles. En revanche, une fois pris en compte les prix des outputs, l'efficacité prix obtenue est moins bonne que celle des exploitations conventionnelles. L'AB n'est pas un gage d'amélioration de la performance économique, ni vraiment de l'efficacité technique pure. Ce résultat diverge de ceux du dossier de l'INSEE «Les exploitations en agriculture biologique : quelles performances économiques?» (Dedieu et al., 2017). En effet, dans cette étude, la viticulture en $\mathrm{AB}$ aurait une meilleure valorisation des produits. Selon les auteurs, les viticulteurs en agriculture biologique génèrent en moyenne un chiffre d'affaires de $17000 €$ par hectare, soit $46 \%$ de plus qu'en conventionnel, notamment grâce à des prix de vente supérieurs (de $10 \%$ à $40 \%$ selon les produits). Malgré des frais de personnel plus d'une fois et demie supérieurs en $A B$, cette meilleure valorisation des vins $\mathrm{AB}$ permettrait d'obtenir un excédent brut d'exploitation (EBE) de $6400 €$ à l'hectare contre $3700 €$ pour les viticulteurs conventionnels. Autrement dit, l'AB générerait plus de richesse et serait plus efficace. Or, pour appuyer ce résultat il faudrait travailler au niveau du résultat courant afin de prendre en compte le coût de l'appareil de production (mesuré par les amortissements annuels) et les frais financiers, ce qui n'est pas le cas.

Dans notre étude, en mesurant l'efficacité prix avec la méthode DEA, nous montrons que la performance économique en $\mathrm{AB}$ n'est pas supérieure aux exploitations conventionnelles, et ce pour différentes raisons qui sont convergentes avec celles du dossier de l'INSEE. Les inputs sont supérieurs ou égaux (intrants, main-d'œuvre et mécanisation) et les rendements sont moindres, et finalement non compensés par la meilleure valorisation économique. Les auteurs du dossier INSEE soulignent d'ailleurs les limites de leur analyse de la performance économique.

\subsection{Limites et perspectives}

Nous sommes conscients des limites de cette étude restreinte à l'analyse de l'efficacité des exploitations viticoles sur deux années. Il est envisagé de traiter la performance des exploitations viticoles d'une série temporelle en données de panel à partir des données du RICA, afin d'évaluer, d'une part, l'évolution de l'efficacité technique pure, et d'autre part, le gain d'efficacité dû aux conséquences de l'adoption de l'innovation et du progrès technique qui changent la fonction de production (Briec et Peypoch, 2010).

L'analyse d'enveloppement des données (DEA) présente des avantages car elle permet avec ces mesures d'efficacité d'ouvrir vers l'analyse des facteurs explicatifs de ces écarts, qu'ils soient technologiques ou institutionnels. Néanmoins, la méthode est fondée sur des conditions strictes comme l'homogénéité des technologies, qui limitent la comparaison entre systèmes de production qui n'auraient pas tout à fait les mêmes technologies. La difficulté réside aussi dans la définition de ce qu'est une technologie identique, tant certains inputs ont des caractéristiques inobservables (potentiel agronomique, conditions pédoclimatiques spécifiques, compétences spécifiques des travailleurs), qui par nature créent de l'hétérogénéité inobservée (Alvarez et al., 2004). Par ailleurs, le choix de la technologie est endogène et l'efficacité, tout comme d'autres co-variables exogènes, affecte la décision du 
choix technologique (Kumbhakar et al., 2009). Nous avions supposé que les viticulteurs en $\mathrm{AB}$ étaient mieux dotés en capitaux (humain, social, économique). Cette hypothèse mériterait d'être discutée à l'avenir, eu égard aux résultats obtenus ici.

Il faut par ailleurs garder à l'esprit que les scores globaux obtenus avec nos traitements masquent la diversité de la performance entre les exploitations AOP (par exemple « Grand Cru », «Village », appellation régionale...) au sein d'une filière AOP et des bassins viticoles. La performance d'une AOP dépend de son cadre institutionnel, de sa gouvernance, de son histoire et des efforts collectifs des différentes parties prenantes (Vandecandelaere et al., 2018). L'étude du Centre d'études et de prospective (CEP) intitulée «Compétitivité hors coût des exploitations agricoles françaises » (Jeanneaux et al., 2018) donne des éclairages sur ces facteurs institutionnels.

Nous savons aussi que l'échantillon basé sur les données RICA n'est pas indemne de critiques. L'échantillon a une taille plus faible que l'étude de l'INSEE à partir de laquelle nous discutons les résultats. Toutefois, nous faisons l'hypothèse que l'échantillon du RICA est représentatif de la viticulture française, même si nous savons que la très grande hétérogénéité des exploitations viticoles est loin d'être prise en compte. Il faudrait pouvoir intégrer un input de caractérisation des terroirs des différents vignobles pour tenter d'approcher l'hétérogénéité spécifique de chaque exploitation. En effet, nos données ne permettent pas par exemple d'intégrer dans l'analyse la spécificité d'une exploitation bourguignonne qui n'aurait que des parcelles en zone AOC premier ou grand cru. Dans notre étude, elle est classée plus largement dans un bassin viticole plus large correspondant à l'appellation régionale.

\section{Conclusion}

Nous nous sommes demandés si trois des signes officiels d'identification de la qualité et de l'origine (AOC/AOP, IGP et $\mathrm{AB}$ ) étaient sources d'une meilleure performance économique et s'ils permettaient d'accroître le revenu des viticulteurs français. Pour ce faire, nous avons analysé les efficacités économiques et techniques des exploitations ayant fait le choix de la différenciation, en évaluant l'efficacité technique pure et l'efficacité prix en traitant les données RICA par la DEA. Nous montrons que les exploitations AOC/AOP et IGP ont des scores d'efficacité plus faibles que celles n'ayant pas adopté ces signes de qualité. La différenciation fondée sur l'origine et le terroir ne semble donc pas être la garantie de l'efficacité économique des exploitations. L'AOC/AOP est devenu un standard de base qui positionne désormais les vins sur le marché de la grande distribution et de l'export à des prix sous pression à la baisse. Autrement dit, les stratégies fondées sur d'autres attributs que l'origine et le terroir ont une meilleure capacité à maximiser les produits pour un niveau d'inputs. Ce résultat est assez inattendu quand on sait que l'AOC/AOP, en dehors de son rôle de protection, est censée créer de la valeur. Le prix joue un rôle clé sur l'efficacité (Delord et al., 2015).

Il ressort par ailleurs que les exploitations en $\mathrm{AB}$ ont des efficacités inférieures aux exploitations conventionnelles. Sur la période étudiée, les efficacités " technique pure» et "prix » des viticulteurs ne sont pas mécaniquement améliorées par l'adoption de cette stratégie. D'autres investigations restent nécessaires pour confirmer ces premiers résultats.

\section{Matériel supplémentaire}

Tableau S1. Description de l'échantillon d'études pour l'année 2014 (Données RICA).

Le matériel supplémentaire est disponible sur http://www. cahiersagricultures.fr/10.1051/cagri/2021036/olm.

Remerciements. Ce travail s'inscrit dans un projet de recherche intitulé «Différenciation», mené en 2017 et 2018 et financé par le Centre d'études et de prospective du Ministère en charge de l'agriculture, dans le cadre de l'appel à projets de recherche «Comprendre et renforcer les compétitivités agricoles, agroalimentaires et forestières ». Les traitements ont été réalisés sur des données accessibles via le Centre d'accès sécurisé aux données (CASD).

\section{Références}

Agence Bio. 2019. L'agriculture biologique, un accélérateur économique, à la résonance sociale et sociétale. Paris (France) : Agence pour le Développement et la Promotion de l'Agriculture Biologique, $43 \mathrm{p}$.

Agence Bio. 2021. Le bio, acteur incontournable de la souveraineté alimentaire. Dossier de presse - juillet 2021 - Les chiffres 2020 du secteur bio. Paris (France): Agence pour le Développement et la Promotion de l'Agriculture Biologique, $28 \mathrm{p}$.

Agra Presse Hebdo. 2004. L'INAO veut distinguer les meilleures AOC. [2020/01/10]. http://www.agrapresse.fr/l-inao-veut-distin guer-les-meilleures-aoc-art227280-5.html.

Alvarez A, Arias C, Greene W. 2004. Accounting for unobservable in production models: Management and inefficiency. Economic Working Paper at Centro de Estudios Andaluces from Centro de Estudios Andaluces, E2004/72, pp.1-18.

Bogetoft P. 2012. Performance analysis DEA. In: Bogetoft P, ed. Performance benchmarking: Mesuring and managing performance. New York (USA): Springer US, pp. 71-102. https://doi.org/ 10.1007/978-1-4614-6043-5 4.

Briec W, Peypoch N. 2010. Microéconomie de la production. Bruxelles (Belgique) : Éditions De Boeck, 245 p.

CCVF. 2015. Bilan des vendages et millésimes 2015. Paris (France) : Confédération des coopératives vinicoles de France, $16 \mathrm{p}$.

Charnes A, Cooper WW, Rhodes E. 1978. Measuring the efficiency of decision-making units. European Journal of Operational Research 2(6): 429-444. https://doi.org/10.1016/0377-2217(78)90138-8.

Dedieu MS, Lorge A, Louveau O, Marcus V. 2017. Les exploitations en agriculture biologique: quelles performances économiques? Paris (France) : Insee Références, $12 \mathrm{p}$.

Delord B, Montaigne E, Coelho C. 2015. Vine planting rights, farm size and economic performance: Do economies of scale matter in the French viticulture sector? Wine Economics and Policy 4(1): 22 34. https://doi.org/10.1016/j.wep.2015.03.001.

Edelmann H, Penker M, Quinones-Ruiz X, Broscha K, Marescotti A, Belletti G, et al. 2020. Social learning in food quality governance? Evidences from geographical indications amendments. International Journal of the Commons 14(1): 108-122. https://doi.org/ 10.5334/ijc.968.

Farrell MJ. 1957. The measurement of productive efficiency. Journal of the Royal Statistical Society 120(3): 253-290. https://doi.org/ $10.2307 / 2343100$. 
FranceAgrimer. 2019. Les chiffres-clés de la filière Viti-Vinicole 2008-2018. Montreuil (France): FranceAgrimer, 168 p.

Guyomard H, ed. 2013. Vers des agricultures à hautes performances, Volume 1: Analyse des performances de l'agriculture biologique. Paris (France) : INRA / Commissariat général à la stratégie et à la prospective, $368 \mathrm{p}$.

Jeanneaux P, Blasquiet-Revol H, Gillot M. 2018 (rendu public en 2019). Compétitivité hors coût des exploitations agricoles françaises. Projet DIFFÉRENCIATION, financé par le ministère de l'Agriculture et de l'Alimentation et VetAgro Sup (UMR Territoires), $119 \mathrm{p}$.

Kumbhakar S, Tsionas M, Sipilainen T. 2009. Joint estimation of technology choice and technical efficiency: An application to organic and conventional dairy farming. Journal of Productivity Analysis 31(3): 151-162. https://doi.org/10.1007/s11123-0080081-y.

Olesen OB, Petersen NC. 2016. Stochastic Data Envelopment Analysis: A review. European Journal of Operational Research 251(1): 2-21. https://doi.org/10.1016/j.ejor.2015.07.058.

Oude Lansink A, Pietola K, Bäckman S. 2002. Efficiency and productivity of conventional and organic farms in Finland 19941997. European Review of Agricultural Economics 29(1): 51-65. https://doi.org/10.1093/erae/29.1.51.
Porter ME. 1985. Competitive advantage. Creating and sustaining superior performance. New York (USA): The Free Press, $32 \mathrm{p}$.

Pouzenc M, Vincq JL. 2013. Faire du bio! Faire du terroir? Le terroir viticole de Gaillac au risque de l'agriculture biologique. Toulouse (France) : Presses Universitaires du Mirail-CNRS, pp. 149-160. https://doi.org/10.4000/soe.519.

Rousset S, Traversac J. 2014. Chapitre 6. Différenciation de la gouvernance territoriale et de la qualité : lecture comparée des filières vitivinicoles. In: Jeanneaux $\mathrm{P}$, ed. Repenser l'économie rurale. Versailles (France) : Éditions Quæ, pp. 94-108. https://doi. org/10.3917/quae.jean.2014.01.0094.

Sahoo BK, Mehdiloozad M, Tone K. 2014. Cost, revenue and profit efficiency measurement in DEA: A directional distance function approach. European Journal of Operational Research 237: 921-931.

Shephard RW. 1981. Cost and production functions. Lecture Notes in Economics and Mathematical Systems. Berlin, Heidelberg: Springer, 106 p. https://doi.org/10.1007/978-3-642-51578-1.

Vandecandelaere E, Teyssier C, Barjolle D, Jeanneaux P, Fournier S, Beucherie O. 2018. Strengthening sustainable food systems through geographical indications-An analysis of economic impacts. Roma (Italy): Nutrition and Food Systems Division and the Investment Centre Division, under the FAO/EBRD cooperation, report $\mathrm{N}^{\circ} 13,135 \mathrm{p}$.

Citation de l'article: Gillot M, Blasquiet-Revol H, Jeanneaux P. 2022. Différenciation et compétitivité : quelle performance pour les exploitations viticoles françaises sous signe de qualité ? Cah. Agric. 31: 2. 